Revista Brasileira de Agricultura Irrigada v.6, n.1, p.51-60, 2012

ISSN 1982-7679 (On-line)

Fortaleza, CE, INOVAGRI - http://www.inovagri.org.br

Protocolo 076.11 - 26/12/2011 Aprovado em 28/03/2012

\title{
ESTIMATIVA DA PRODUTIVIDADE DE ÁGUA EM UMA ÁREA IRRIGADA NO SUL DA ESPANHA
}

\author{
Sílvio Carlos Ribeiro Vieira Lima ${ }^{1}$; José Antônio Frizzone² ${ }^{2}$ Luciano Mateos $^{3}$; \\ Maria Sierra Fernandez ${ }^{4}$
}

\section{RESUMO}

O Projeto de Irrigação Genil-Cabra (PIGC), Coletividade Santaella, está localizado na província de Córdoba-Espanha. Nesse Distrito avaliou-se a irrigação das campanhas de 2004/2005 e 2005/2006. Após a coleta dos dados, foram determinados indicadores da eficiência da irrigação na área. pela metodologia utilizada por Mateos (2007). Como complementação a essa metodologia, foram introduzidas outras ferramentas, como Modelo Digital do Terreno - DEM, Sistema de Informação Geográfica - SIG e entrevistas com os agricultores. Depois de entrevistar os agricultores e de comparar as informações com as ortofotos obtidas pelo site de cadastro, foi possível determinar a produtividade da água (WP). Observou-se que os maiores valores foram detectados pela análise por recinto, tanto em 2005 quanto em 2006, justificadas pelo baixo volume de água demandado, observado nos sensores. No cálculo para a WP na parcela, usa-se, como denominador, a evapotranspiração da cultura, enquanto, no cálculo da WP do recinto, usa-se o volume de água que entra na área, ou seja, a água aplicada. Visto que a irrigação foi deficitária, então certamente haverá uma maior produtividade da água no nível do recinto. Os valores obtidos foram 4,06;0,37€ m-3 para recintos e parcelas (2005) e 6,60;0,27€ m-3 (2006). Esses valores são similares aos encontrados por Garcia-Vila et al. (2008) e Lorite et al. (2004b).

Palavras-chave: Produtividade de Água; Balanço de água; Irrigação deficitária

\section{ESTIMATION OF THE WATER PRODUCTIVITY ON THE IRRIGATED AREA IN SOUTHERN SPAIN}

\footnotetext{
ABSTRACT

The Genil-Cabra Irrigation Project (PIGC), Coletividade Santaella, is located in Andalusia, southern Spain. It was evaluated the irrigation in 2004/2005 and 2005/2006 seasons. After collecting all data it was made a study to determine indicators to irrigation efficiency for methodology used by Mateos (2007) complemented with the Digital Terrain Model - DEM, Geographic Information System - SIG and farmer interviews. After the farmer interviews and compared the information with the

${ }^{1}$ Pesquisador do Instituto INOVAGRI, silviocarlos@inovagri.org.br.

${ }^{2}$ Professor Titular, ESALQ/USP .

${ }^{3}$ Investigador Científico, IAS/CSIC - Córdoba -Espanha.

${ }^{4}$ Engenheira Agrônoma, Córdoba -Espanha.
} 


\section{ESTIMATIVA DA PRODUTIVIDADE DE ÁGUA EM UMA ÁREA IRRIGADA NO SUL DA ESPANHA}

orthophotos obtained from the web's records it was possible determine the water productivity (WP). It was observed that the biggest values were detected by "recinto" analyze, both in 2005 and 2006 season, due to low water volume demanded, observed in the sensors. In the parcel WP calculation, it was used the crop evapotranspiration as denominator; however in the "recinto" WP calculation it was used the applied water. Since the irrigation was deficiency, then likely will have a bigger WP in the "recinto" level. The values obtained were 4.06;0.37(€ m-3) to "recintos" and parcels (2005) and $6.60 ; 0.27(€ \mathrm{~m}-3)$ to "recintos" and parcels (2006). These results are similar to encountered by Garcia-Vila et al. (2008) and Lorite et al. (2004b).

Keywords: Water productivity; Water balance; Deficit Irrigation.

\section{INTRODUÇÃO}

$\mathrm{Na}$ Espanha, a Comunidade Santaella, localizada no Projeto de Irrigação Genil-Cabra (PIGC), começou a atuar na campanha de irrigação de 1990/1991 em uma área de 2.663 ha, ampliada para cerca de 6.900 ha em 1993/1994, e que se mantém constante, ocupando $45 \%$ da área do PIGC, onde trabalham $47 \%$ dos agricultores (IFAPA, 2005).

Fernandez et al. (2007) citado por Lima et al (2010) avaliaram o desempenho da irrigação e a contaminação difusa dessa área por agroquímicos, durante a campanha de 2005/2006, utilizando a metodologia de Duarte (2006), aplicada em Portugal. Os autores não obtiveram conclusões satisfatórias para a contaminação difusa, porém verificaram que a área utiliza irrigação deficitária com alto índice de eficiência.

As práticas convencionais de irrigação baseiam-se na necessidade de água da cultura, definida pela demanda evapotranspirométrica, e na eficiência da aplicação da água. (FRIZZONE, 2007). De acordo com o autor, na irrigação com déficit, planeja-se atender somente a uma fração da demanda de água da cultura por evapotranspiração. Esse tipo de manejo pode ser praticado com irrigação total e com irrigação suplementar. O déficit de água pode ser imposto durante todo o ciclo da cultura, ou somente nas fases não-críticas ao déficit hídrico.

A irrigação com déficit tem sido amplamente investigada como uma estratégia valiosa para as regiões secas (ENGLISH, 1990; FERERES; SORIANO, 2007; MATEOS, 2008) onde a água é o fator limitante no cultivo.

O potencial de retorno de irrigação com déficit deriva de três fatores: aumento da eficiência da irrigação, redução de custos da irrigação e o custo de oportunidade da água (ENGLISH et al., 1990 citado por ALI et al., 2007).

A irrigação com déficit é sucesso no aumento da produtividade da água para diversas culturas sem causar reduções de rendimento grave. Esta técnica exige um conhecimento exato da resposta da cultura ao estresse hídrico, pois a tolerância à seca varia consideravelmente pelo genótipo e estádio fenológico da planta.

Playán e Mateos (2006) verificaram que, em grandes e antigos distritos de irrigação da Espanha, foram incentivadas técnicas de manejo para reduzir o consumo excessivo de água de determinadas culturas; como resultado, obtiveram uma considerável economia de água, com praticamente a mesma produtividade das culturas. Isso apenas com a orientação e a reeducação dos agricultores. 


\section{ESTIMATIVA DA PRODUTIVIDADE DE ÁGUA EM UMA ÁREA IRRIGADA NO SUL DA ESPANHA}

Indicadores de desempenho têm sido um componente da prática de irrigação, desde que o homem começou a controlar a água para aumentar a produção de alimentos. Os antigos egípcios monitoravam o nível de água do rio Nilo para saber qual área poderia ser irrigada, de modo a prevenir-se de enchentes e da falta de alimentos (BOS et al., 1994).

Segundo Perry et al. (2009), para entender melhor como as culturas, os ambientes agroclimático e as diferentes práticas de manejo podem influenciar na relação entre a produção agrícola e o consumo de água, pode-se definir a produtividade de água para uma cultura (WP) como a razão entre a quantidade produzida e a quantidade de água consumida para obter essa produção. Além disso, a definição da produtividade da água precisa ser específica, indicando qual o produto (ou o rendimento da biomassa) e qual o consumo por transpiração (ou evapotranspiração).

Ali e Talukder (2008) apresentam uma síntese do incremento em produtividade da água na agricultura e concluem que novos dados científicos são necessários para melhorar os ganhos econômicos de WP porque as melhorias futuras em WP parecem ser mais limitadas por razões econômicas do que pela falta de meios tecnológicos.

\section{MATERIAL E MÉTODOS}

Esta pesquisa seguiu a metodologia utilizada por Mateos (2007) para avaliar a irrigação deficitária.

A necessidade de refinar $o$ trabalho de sistema de informação geográfica realizado por Fernandez et al. (2007), foi de fundamental importância para o início da avaliação da área, tendo sido necessária a
Mateos (2007) desenvolveu metodologia para avaliar os indicadores de desempenho da irrigação, em condições de déficit hídrico, a qual está sendo utilizada no PIGC, assim como é utilizada em diversas pesquisas na Espanha, principalmente em áreas irrigadas às margens do Mediterrâneo. A base da metodologia é a realização do balanço hídrico climatológico, para determinar a evapotranspiração das culturas em condições deficitárias de irrigação.

De posse de dados da irrigação pré-existentes de uma área irrigada, foram avaliadas a irrigação das campanhas de 2004/2005 e 2005/2006. Como complementação ao trabalho, foi utilizado um modelo digital de terreno, produzido pelo governo da Andaluzia, por meio do qual foi possível definir uma melhor delimitação da área que contribuiria com o estudo; um novo sistema de informação geográfica também foi elaborado e, para complementar a análise, foram realizadas entrevistas com os agricultores da área estudada, objetivando determinar os custos de produção na área, a adoção das novas tecnologias por parte da comunidade, a orientação do Sistema de Assessoramento ao Irrigante - SAR a produtividade e o consumo da água em cada parcela.

complementação com as técnicas de geoprocessamento para organizar os dados. Nesta fase foram utilizados softwares como o ArcGis 9.1 e softwares livres como o Kosmos 2.1.

A base de dados foi reformulada com informações dos agricultores, novas delimitações de área oriundos do novo DEM e os dados do balanço de água no solo. Após a alimentação dos 


\section{ESTIMATIVA DA PRODUTIVIDADE DE ÁGUA EM UMA ÁREA IRRIGADA} NO SUL DA ESPANHA

dados no SIG, foi feito o geoprocessamento e gerados os mapas, utilizando as ferramentas dos softwares.

Depois de avaliado, com acompanhamento in loco, o manejo da irrigação, bem como analisados os dados dos anos anteriores e definida a eficiência da irrigação e os demais indicadores de uso da água, foram introduzidas as avaliações sobre indicadores econômicos e sociais conforma Lima (2010).
Para iniciar essa avaliação ao longo das campanhas 2004/2005 e 2006/2007, por meio de dados obtidos e de estudos já realizados, definiu-se a evolução dos indicadores econômicos de produtividade de água, na parcela, bem como no recinto (aglomeração de mais de uma parcela de onde a água está sendo derivada) de acordo com Equação 01, conforme Malano e Burton (2001) citados por Lorite et al. (2004):

$$
\text { WP }\left(€ \mathrm{~m}^{-3}\right)=\frac{\text { Valor anual da produçãoagrícola }}{\text { Volume anual de irrigação }}
$$

$\mathrm{Na}$ avaliação da produtividade de água no nível da parcela, foi definida a equação (02) e o do recinto, a equação
(03) Malano e Burton (2001) citados por Lorite et al. (2004).

$$
\begin{aligned}
& \text { WP }\left(€ \mathrm{~m}^{-3}\right)=\frac{\text { Valor anual da produçãoagrícola }}{\text { Evapotranspiração da cultura no período }} \\
& \mathrm{WP}\left(€ \mathrm{~m}^{-3}\right)=\frac{\text { Valor anual da produçãoagrícola }}{\text { Volume de água observado no contador }}
\end{aligned}
$$

No trabalho realizado por Fernandez et al. (2007), a área foi dividida em 17 parcelas, denominadas recintos. Após a realização da entrevista, identificou-se a necessidade de dividi-la por áreas de cadastro,

\section{RESULTADOS E DISCUSSÃO}

Na Figura 01 é observada a delimitação da bacia, sobre uma ortofoto e após o SIG final. Na Figura 02, temos a nova área com delimitação por cadastros após as devidas correções permitidas pelo acesso ao site de cadastro e a entrevista com os irrigantes. Nota-se que a área de cadastro difere sempre da área de recinto Figura 03. Na Figura 04 são observados os cultivos presentes na área, no ano de 2005, enquanto, na identificadas pelo produtor e pelo site oficial de cadastro. Dessa forma, a área, após o SIG final, passou a ter 33 parcelas, dando uma maior amplitude à pesquisa.

Figura 05, no ano de 2006. A razão é que a denominação recinto está relacionada à tomada de água da parcela, que pode até mesmo ter proprietários diferentes e quase sempre cultivos distintos em um mesmo recinto. Importante também é relatar que, após a entrevista com os agricultores, comparando-se as informações com as ortofotos obtidas pelo site de cadastro, puderam-se identificar cultivos diferenciados dos citados por Fernandez et al. (2007). O fato foi importante para definir a eficiência do uso da água, já que a área 


\section{ESTIMATIVA DA PRODUTIVIDADE DE ÁGUA EM UMA ÁREA IRRIGADA NO SUL DA ESPANHA}

com oliveira (Olea europaea L.) foi reduzida (LIMA, 2010). Na campanha de 2005, este cultivo ocupou uma extensão de 105,61 ha. Já na campanha de 2006, com o cultivo de novas áreas com oliveiras, esse valor passou para 112 ha, que contrastam com os 169,25 ha calculados na delimitação anterior feita por Fernandez et al. (2007). Acredita-se que essa diferença, além da nova delimitação feita pelo DEM, devese também à entrevista realizada com os produtores que de precisão aos dados.

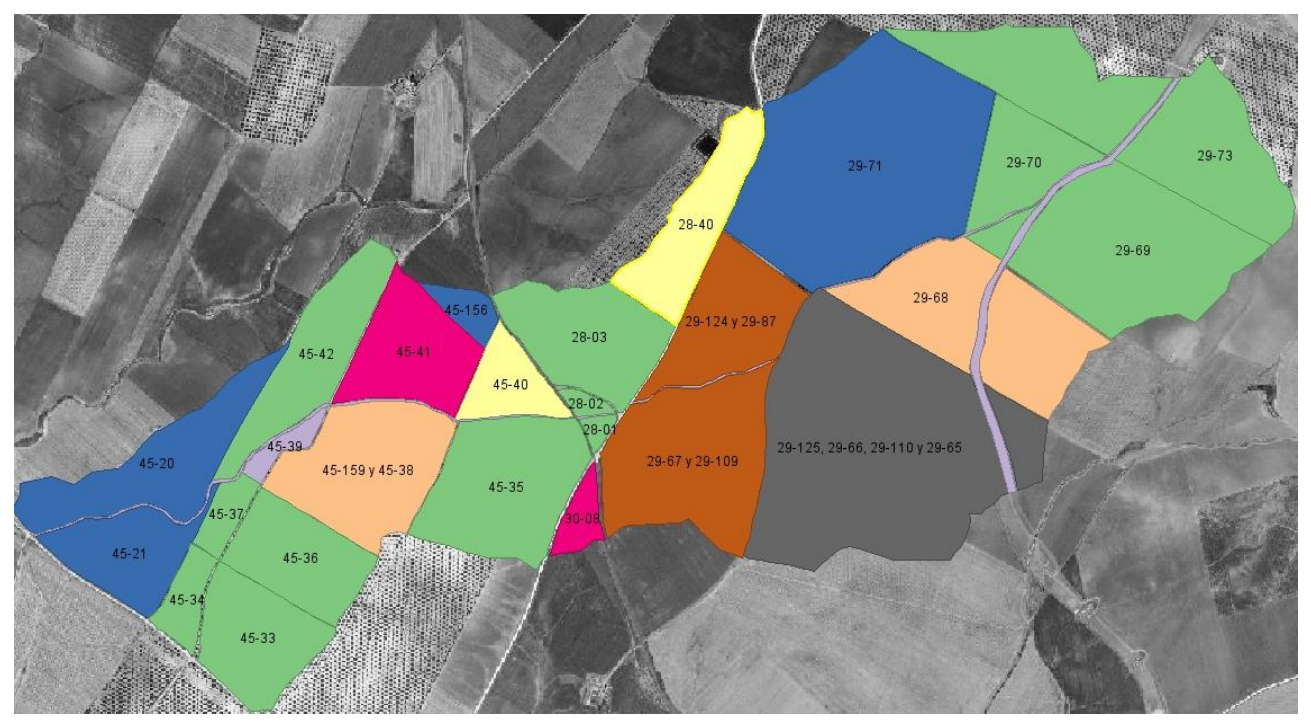

Figura 01 - Área da bacia sobre a ortofoto

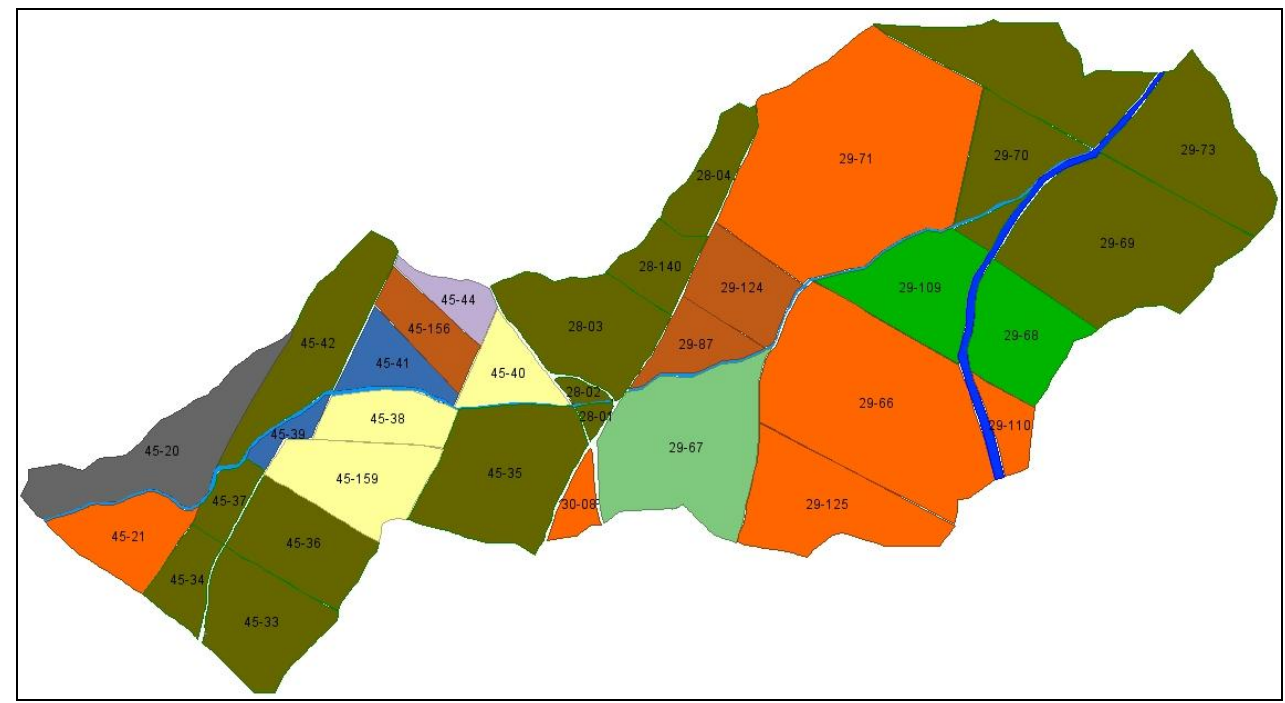

Figura 02 - Nova área com delimitação por Cadastros 
ESTIMATIVA DA PRODUTIVIDADE DE ÁGUA EM UMA ÁREA IRRIGADA

NO SUL DA ESPANHA

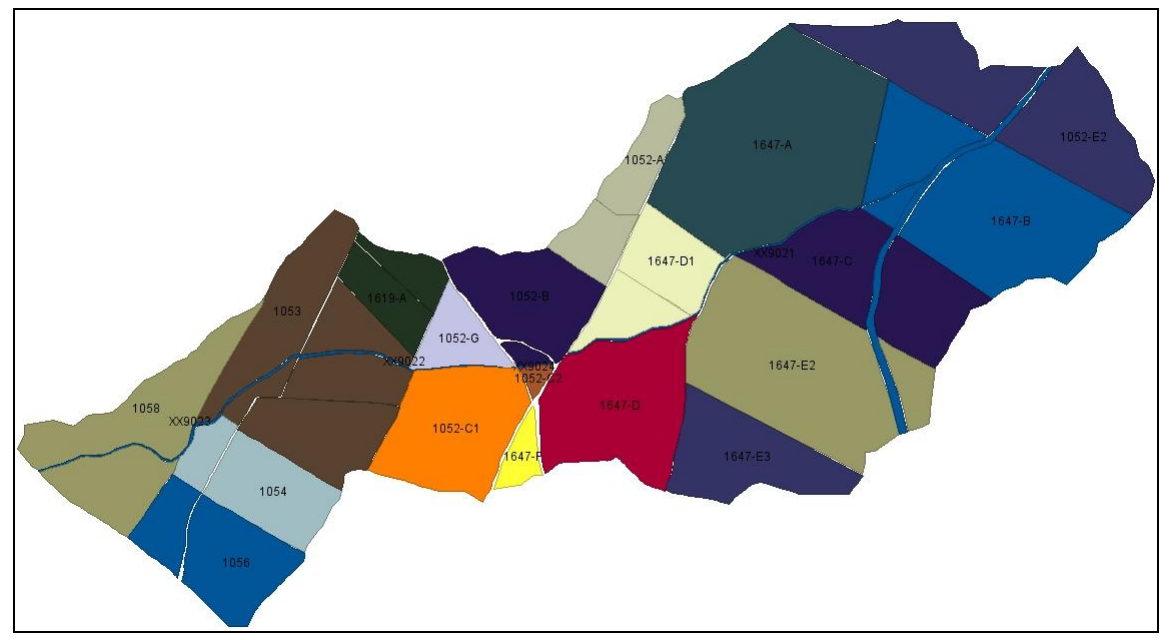

Figura 03 - Nova área por delimitação de Recintos

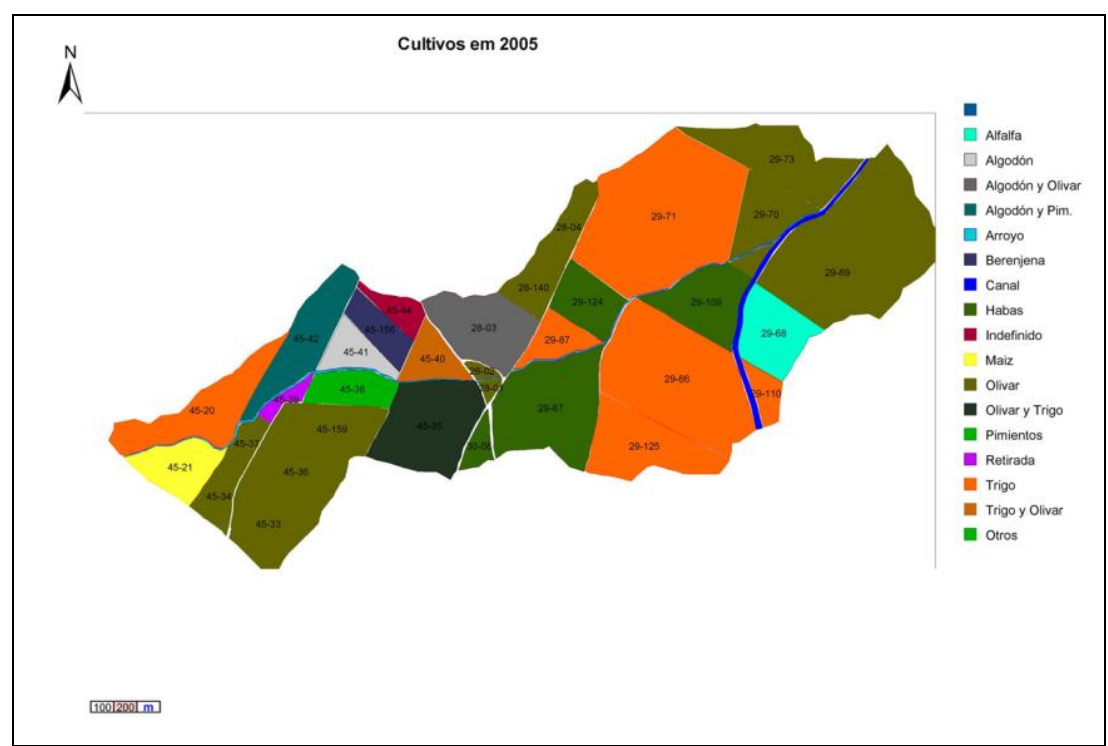

Figura 04 - Nova área da bacia com cultivos de 2005

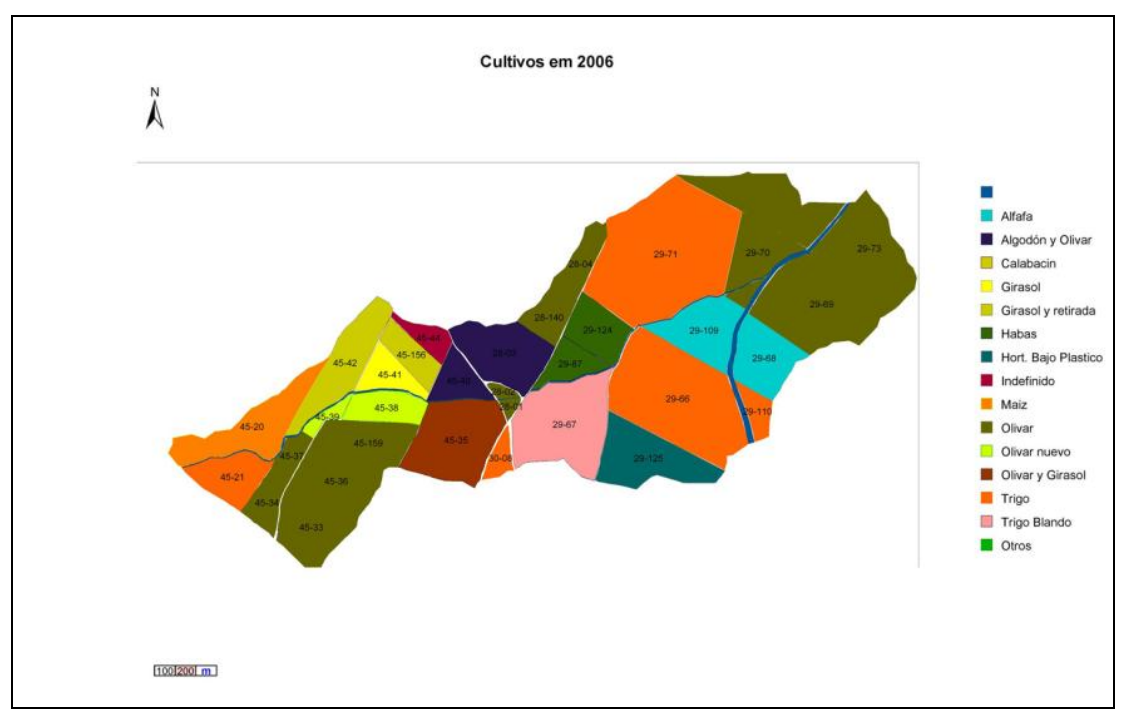

Figura 05 - Nova área da bacia com cultivos de 2006 


\section{ESTIMATIVA DA PRODUTIVIDADE DE ÁGUA EM UMA ÁREA IRRIGADA} NO SUL DA ESPANHA

No cálculo para WP na parcela tem-se como denominador a evapotranspiração da cultura e no cálculo da WP do recinto, tem-se o volume de água que entrou na área, ou seja, a água aplicada. Visto que a irrigação foi deficitária, então certamente haverá uma maior produtividade de água ao nível de recinto.

Os valores encontrados de WP neste trabalho podem ser visualizados na Tabela 01 e representados espacialmente pelas Figuras 06, 07, 08 e 09 . Valores acima de $2 € \mathrm{~m}^{-3}$ são muito encontrados quando se calcula a WP Recinto. Para este indicador, no ano de 2005 foram identificados 08 valores dos 29 disponíveis. Já para a WP Parcela neste mesmo ano, apenas 01 valor foi encontrado. Em praticamente todos os casos, foi com a cultura da oliveira. As exceções foram alfafa e algodão. No ano de 2006, para WP Recinto, foram observados valores em 09 análises. Para WP Parcela, novamente apenas 01 para a cultura do milho. A alfafa, novamente, apresentou valores de WP elevados neste ano. Uma surpresa foi o trigo, que no ano de 2005 teve baixa WP.

Em linhas gerais, os resultados observados são similares aos encontrados por Garcia-Vila et al. (2008). Segundo estes autores, que estudaram dados de 15 anos de algumas parcelas deste projeto de irrigação, a produtividade de água (valor monetário da produção dividido pelo volume de água de irrigação derivada), foi moderada e altamente variável (em torno de $2 € \mathrm{~m}^{-3}$ ) e não houve incremento com o tempo. Já com relação à produtividade da água de irrigação (aumento do valor monetário da produção devido à irrigação, dividida pela água de irrigação entregue) foi bem menor $\left(0,65 € \mathrm{~m}^{-3}\right) \mathrm{e}$, também, não houve incremento com o tempo.

A variação entre WP de parcelas foi bastante diversificada entre cultivos o que leva a crer que dada a ampla gama de utilização da água e de gestão encontrada nas parcelas estudadas, a melhoria da gestão de uma área irrigada deve sempre considerar desempenho individual de cada agricultor ao conceber medidas para a conservação da água na agricultura irrigada, o que também foi ressaltado por Lorite (2004b), estudando o mesmo projeto de irrigação, em que se deduziu que a produtividade da água foi mais elevada para culturas hortícolas e frutíferas como o alho e a oliveira cujo valor variou de $1,13 € \mathrm{~m}^{-3}$ para $6,52 € \mathrm{~m}^{-3}$, respectivamente. Em outras culturas extensivas ocorreu uma variação menor como no caso do milho $\left(0,28 € \mathrm{~m}^{-3}\right)$ e mais elevada na beterraba $\left(1,04 € \mathrm{~m}^{-3}\right)$.

\section{CONCLUSÕES}

Após determinar a produtividade da água (WP), observou-se que os maiores valores foram detectados pela análise por recinto, tanto em 2005 quanto em 2006, justificadas pelo baixo volume de água demandado. Visto que a irrigação foi deficitária, então certamente haverá uma maior produtividade da água no nível do recinto.

A metodologia de Mateos (2007) é valida pode ser alvo de aplicação em outras áreas irrigadas brasileiras.

Tabela 01. Produtividade de água para as campanhas de 2004/2005 e 2005/2006

\begin{tabular}{|c|c|c|c|c|}
\hline Campanha & $\begin{array}{c}\text { Área de } \\
\text { Recinto }\left(\mathbf{m}^{2}\right)\end{array}$ & $\begin{array}{l}\text { Área cultivada } \\
\left(\mathrm{m}^{2}\right)\end{array}$ & $\begin{array}{c}\text { PW }\left(€ \mathrm{~m}^{-3}\right) \\
\text { Recinto }\end{array}$ & $\begin{array}{c}\mathbf{P W}\left(€ \mathrm{~m}^{-3}\right) \\
\text { Parcela }\end{array}$ \\
\hline $2004 / 2005$ & 4.463 .707 & 2.979 .133 & 4,06 & 0,37 \\
\hline $2005 / 2006$ & 4.463 .707 & 2.979 .133 & 6,18 & 0,27 \\
\hline
\end{tabular}




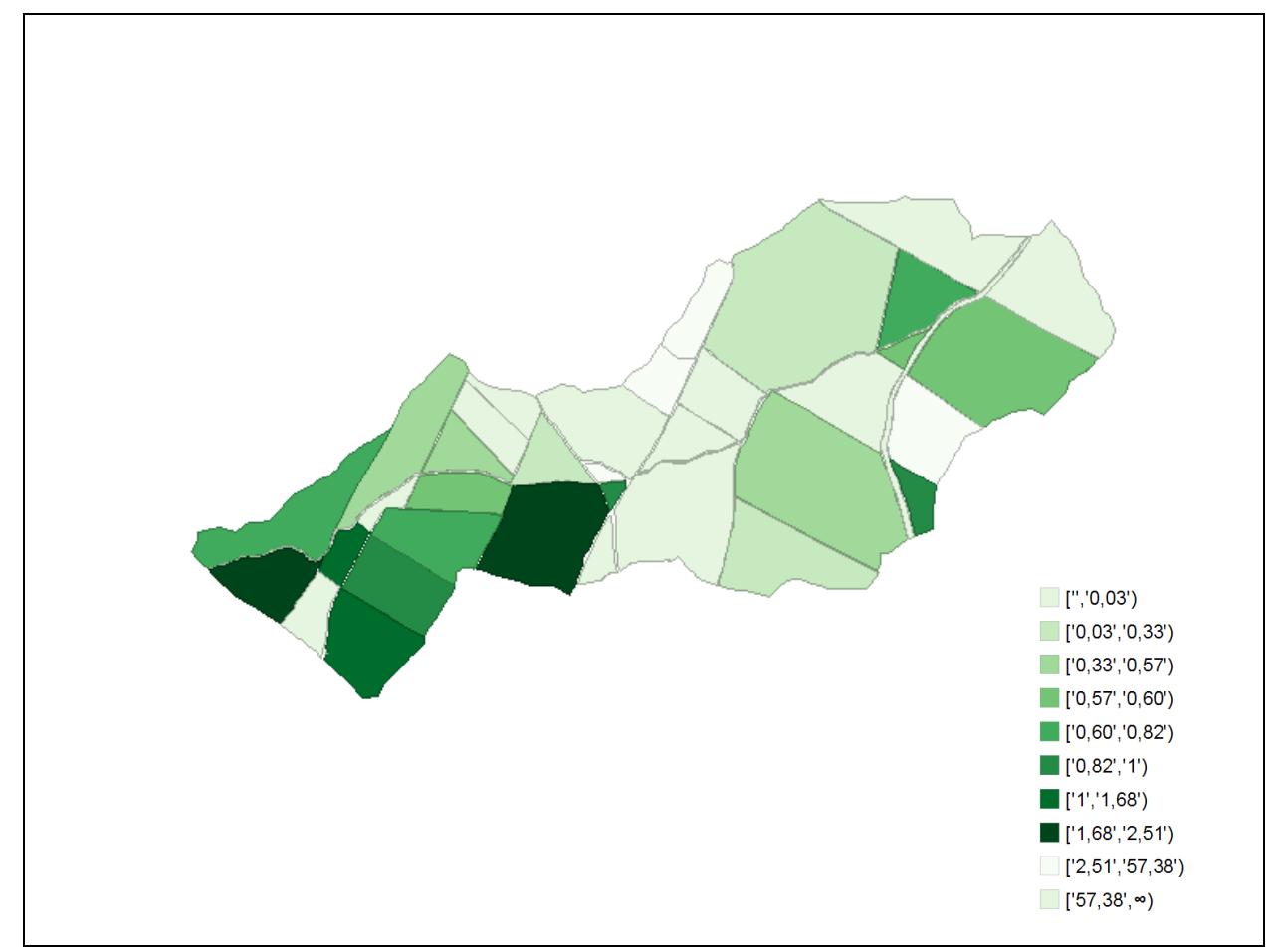

Figura 06 - Produtividade da água $\left(€ \mathrm{~m}^{-3}\right)$ por parcela 2004/ 2005

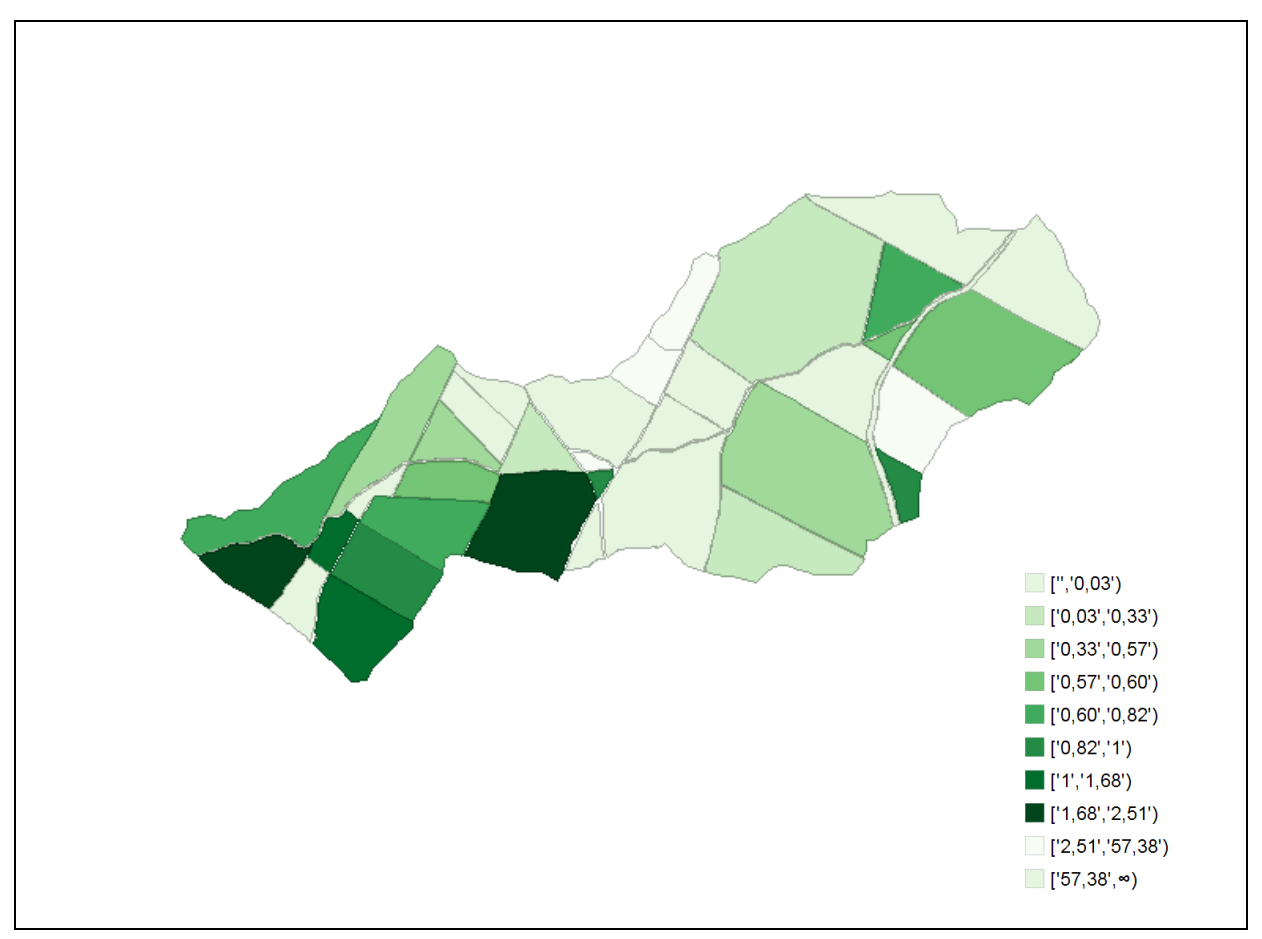

Figura 07 - Produtividade da água $\left(€ \mathrm{~m}^{-3}\right)$ por recinto $2004 / 2005$ 


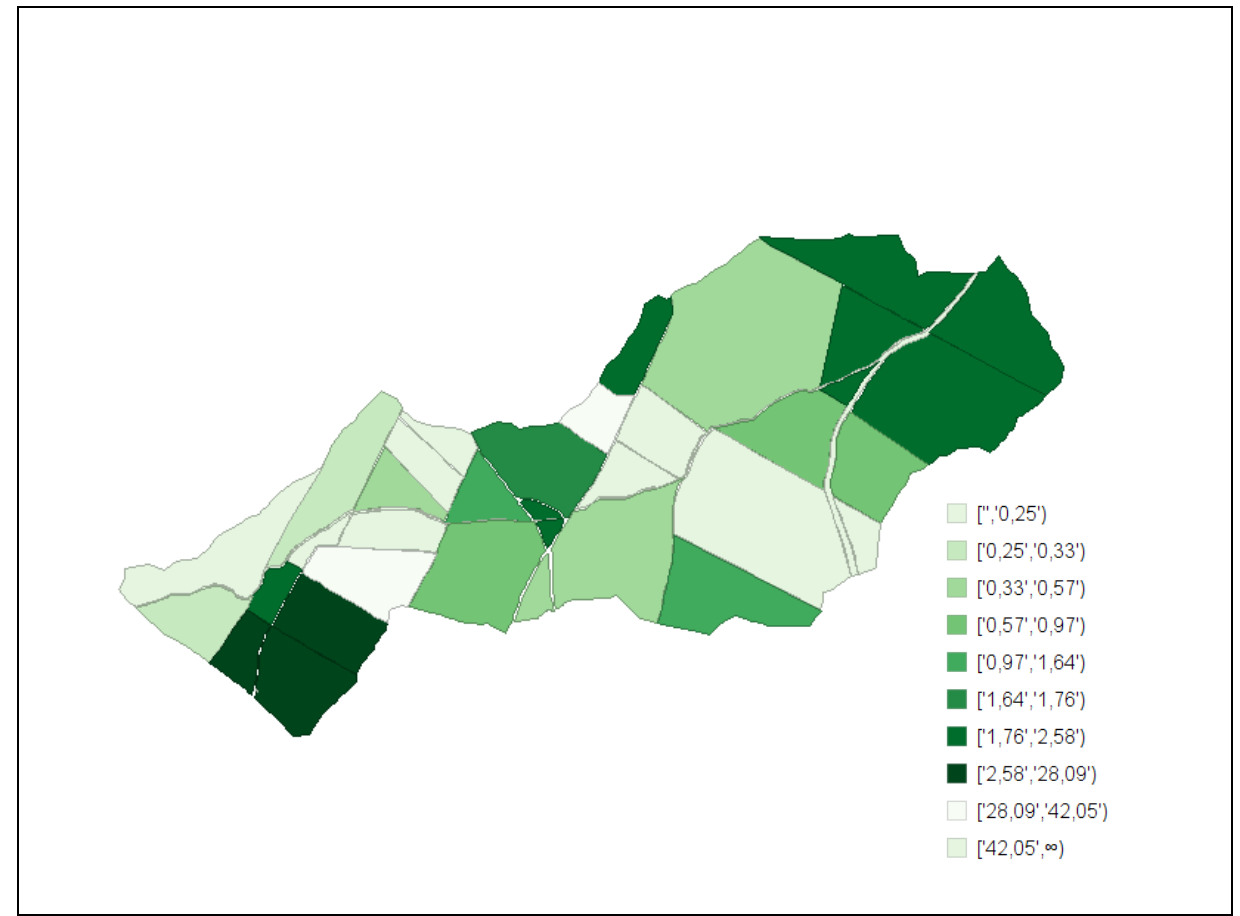

Figura 08 - Produtividade da água $\left(€ \mathrm{~m}^{-3}\right)$ por parcela 2005/2006

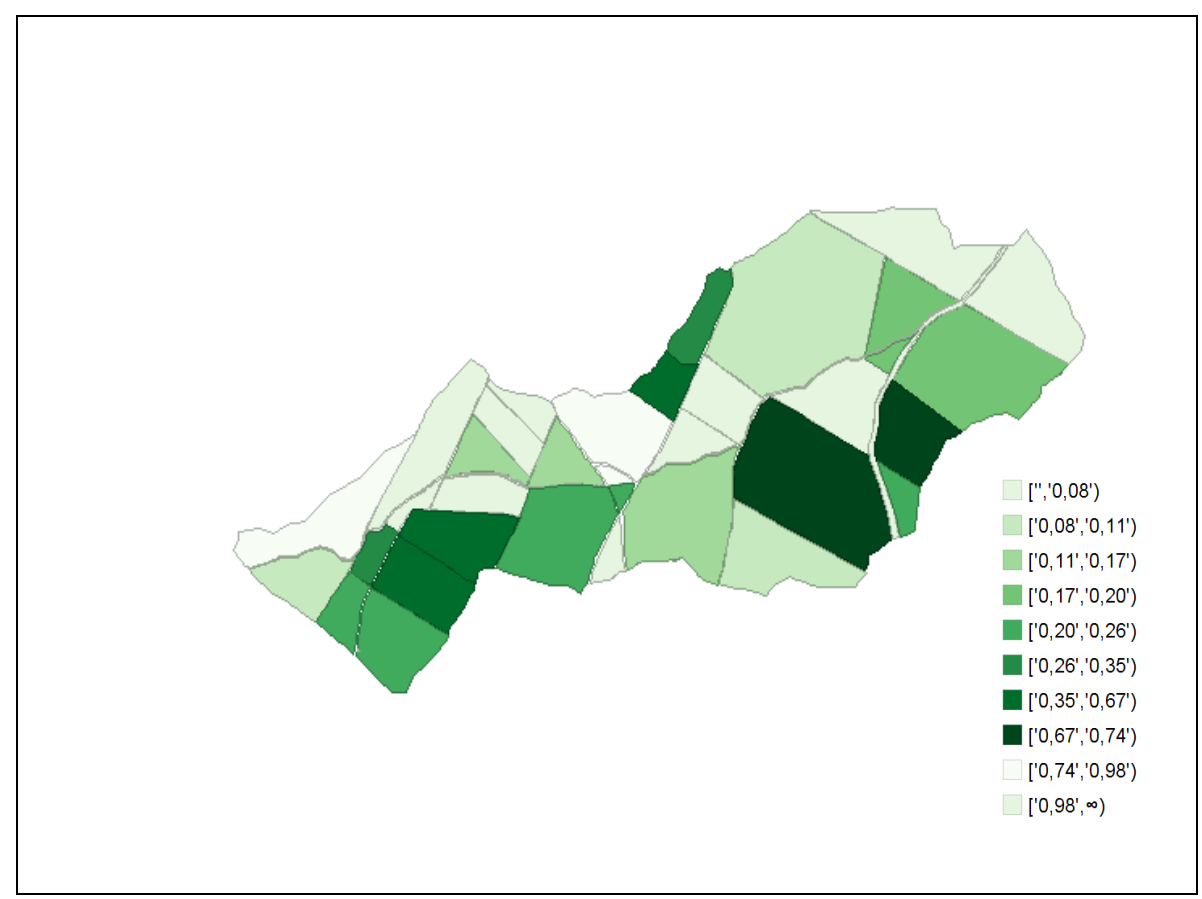

Figura 09 - Produtividade da água $\left(€ \mathrm{~m}^{-3}\right)$ por recinto no 2005/2006

\section{REFERÊNCIAS}

ALI M.H.; TALUKDER, M.S.U. Increasing water productivity in crop production - A synthesis
Agricultural Water Management, Amsterdam, v. 95 p. 201-213, 2008. BOS, M.G.; MURRAY-RUST, D.H.; MERREY, D.J.; JOHNSON, H.G.; SNELLEN, W.B. Methodologies for assessing performance of irrigation 


\section{ESTIMATIVA DA PRODUTIVIDADE DE ÁGUA EM UMA ÁREA IRRIGADA} NO SUL DA ESPANHA

and drainage management. . Irrigation and Drainage Systems, Dordrecht, v. 7, p. 231-261, 1994.

DUARTE,

A.F.C.

Contaminación difusa originada por la actividad agrícola de riego, a escala de cuenca hidrográfica. 2006. 219 p. Tesis (Doctoral) - Escuela Técnica Superior de Ingenieros Agrónomos y de Montes, Universidad de Córdoba, Córdoba, 2006.

FERERES, E.; SORIANO, M.A. Deficit irrigation for reducing agricultural water use. Special issue on 'Integrated approaches to sustain and improve plant production under drought stress' Journal of Experimental Botany, Lancaster, v. 58, p.147-159, 2007.

FERNANDEZ, M. S. F.; ASCENSIÓN, M.A.C.; MATEOS, L.; FERERES E. Comportamiento del riego a la escala de una pequeña cuenca In: MATEOS, L. Metodologías e instrumentos para a planificación y la gestión sostenible en condiciones de escassez de agua. Córdoba: CSIC, 2007. p. 31-50.

FRIZZONE, J.A. Planejamento da irrigação com uso de técnicas de otimização. Revista Brasileira de Agricultura Irrigada, Fortaleza, v. 1, n. 1, p. 24-49, dez. 2007.

GARCÍA-VILA M., I.J.; LORITE, M.A.; SORIANO, F.E. Management trends and responses to water scarcity in an irrigation scheme of Southern Spain. Agricultural Water Management, Amsterdam, v. 95, p. 458-468, 2008.

INSTITUTO ANDALUZ DE INVESTIGACIÓN Y FORMACIÓN AGRARIA, PESQUERA, ALIMENTARIA Y DE LA PRODUCCIÓN ECOLÓGICA, Unidad de apoyo técnico al regadío andaluz.
Córdoba, 2005. 10 p. (IFAPA Boletín Trimestral de Información, 1).

LIMA, S. C. R. V. ; FRIZZONE, J. A. ; MATEOS, L. ; FERNANDEZ. M. S. . Desempenho da Irrigação em um Projeto Hidroagrícola no Sul da Espanha: metodologia para análise da eficiência de uso da água. Revista Brasileira de Agricultura Irrigada, v. 4, p. 59-77, 2010.

LORITE, I.J.; MATEOS, L.; FERERES, E. Evaluating irrigation performance in a Mediterranean environment. I. Model and general assessment of an irrigation scheme. Irrigation Science, Amsterdam, v. 23, p. 77-84, 2004a.

performance ${ }^{-}$Evaluating in a Mediterranean environment. II. Variability among crops and farmers. Irrigation Science, Amsterdam, v. 23, p. 85-92, 2004b.

MATEOS, L. Metodología "mipais" para el riego en condiciones de escasez de agua In:

Metodologías e instrumentos para a planificación y la gestión sostenible en condiciones de escassez de agua. Córdoba: IAS/CSIC, 2007. cap. 4, p. 25-30.

Identifying a new paradigm for assessing irrigation system performance Irrigation Science, Amsterdam, v. 27, p. 25-34, 2008.

PERRY, C.; STEDUTO, P.; ALLEN, R.G.; BURT, C.M. Increasing productivity in irrigated agriculture: Agronomic constraints and hydrological realities. Agricultural Water Management, Amsterdam, v. 96, p.1517-1524, 2009.

PLAYÁN, E.; MATEOS, L. Modernization and optimization of irrigation systems to increase water productivity Agricultural Water Management, Amsterdam, v. 80, p. 100-116, 2006. 